\title{
Drop Fragmentation at Impact onto a Bath of an Immiscible Liquid
}

\author{
H. Lhuissier, ${ }^{*}$ C. Sun, A. Prosperetti, and D. Lohse \\ Physics of Fluids Group, Faculty of Science and Technology, MESA ${ }^{+}$Institutes, and Burgers Center for Fluid Dynamics, \\ University of Twente, 7500AE Enschede, The Netherlands \\ (Received 30 October 2012; published 28 June 2013)
}

\begin{abstract}
The impact of a drop onto a deep bath of an immiscible liquid is studied with emphasis on the drop fragmentation into a collection of noncoalescing daughter drops. At impact the drop flattens and spreads at the surface of the crater it transiently opens in the bath and reaches a maximum deformation, which gets larger with increasing impact velocity, before surface tension drives its recession. This recession can promote the fragmentation by two different mechanisms: At moderate impact velocity, the drop recession converges to the axis of symmetry to form a jet which then fragments by a Plateau-Rayleigh mechanism. At higher velocity the edge of the receding drop destabilizes and shapes into radial ligaments which subsequently fragment. For this latter mechanism the number $N \propto \mathrm{We}^{3}$ and the size distribution of the daughter drops $p(d) \propto d^{-4}$ as a function of the impact Weber number We are explained on the basis of the observed spreading of the drop. The universality of this model for the fragmentation of receding liquid sheets might be relevant for other configurations.
\end{abstract}

DOI: 10.1103/PhysRevLett.110.264503

A drop impacting onto a deep liquid bath is well known to transiently open a crater in the bath and possibly eject a liquid sheet and a jet. Since Worthington's drawings and photographs more than a century ago [1,2], much attention has been paid to the impact with identical or miscible drop and bath liquids. Although the long term state itself, the coalescence of the drop, was not an issue, the description of the transient structures [3-6] was motivated by the possible ejection of drops in the atmosphere, bubble entrainment [7,8], and the underwater noise of the rain [9]. By contrast, we study here the impact of a drop onto an immiscible bath with particular focus on the nontrivial final state: the dispersion of the impacting drop into a collection of noncoalescing daughter drops. This configuration has applications for many industrial processes, among which: metallic melt quenching [10], accidental release of metallic liquid into hot pools [11] or drop encapsulation by immersion into a reacting liquid bath [12]. We first describe the different fragmentation behaviors that are observed for an increasing impact velocity and then focus on the high velocity regime for which both the number of daughter drops and the universal structure of their size distribution are derived.

Our experiment consists of a water drop (with density $\rho$ and surface tension $\sigma$ [13]), impacting perpendicularly onto the horizontal surface of a bath of silicone oil. Four different silicone oils with viscosities $\nu_{B}=0.65,10,50$, and $200 \mathrm{~mm}^{2} \mathrm{~s}^{-1}$ are used [13]. Both liquids are immiscible. The drop is formed in a quasistatic way at the tip of a vertical syringe needle. It detaches by gravity at a reproducible size $d_{0}=2.92 \pm 0.03 \mathrm{~mm}$, which is measured by weighing the drop and kept constant for all the experiments, and then falls perpendicularly onto the bath surface. The drop velocity $V$ is adjusted from the height of the needle and
PACS numbers: 47.20.Ma, 47.20.Ky, 47.55.D-, 47.55.nb

measured just before impact within $1 \%$ error with a fast camera.

Figure 1 illustrates the impact subsurface dynamics for increasing velocities, corresponding to $\mathrm{We}=21,205$, and 305 , respectively, where $\mathrm{We}=\rho V^{2} d_{0} / \sigma$ is the Weber number which compares the kinetic to the surface energy of the drop. At impact the drop sets the surrounding bath liquid into motion and opens a crater. This crater eventually reaches a maximal size, where the flow in the bath essentially vanishes, and then collapses, driven by buoyancy forces. At the same time, the drop flattens and spreads just below the crater surface in a way reminiscent of the impact of a drop onto a solid $[14,15]$ or even more onto a

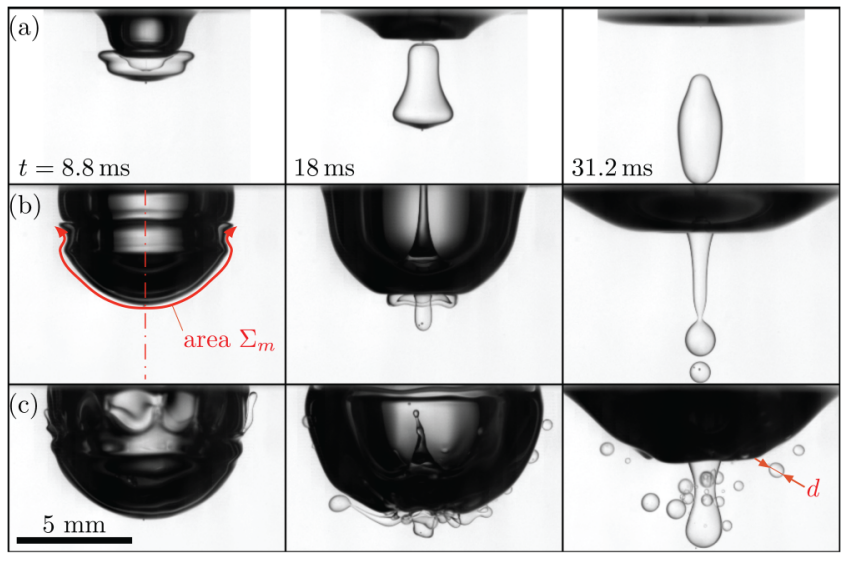

FIG. 1 (color online). Subsurface view of a water drop $\left(d_{0}=2.92 \mathrm{~mm}\right)$ impact onto a deep silicone oil bath $\left(\nu_{B}=10 \mathrm{~mm}^{2} \mathrm{~s}^{-1}\right)$ showing the drop recession after spreading, for increasing impact velocities: (a) $V=0.73 \mathrm{~m} \mathrm{~s}^{-1}$, (b) $V=2.26 \mathrm{~m} \mathrm{~s}^{-1}$, (c) $V=2.96 \mathrm{~m} \mathrm{~s}^{-1}$. For each sequence, the images are taken at the same time $t$ after the first contact. 
deformable surface [16]. Surface tension then drives the recession of the flattened drop and depending on the impact velocity, three different regimes, respectively illustrated in Figs. 1(a)-1(c), are observed: (1) At low velocity the drop does not fragment. (2) At moderate velocity the drop shapes into a single ligament and fragments into only a few droplets along the (vertical) axis of symmetry. (3) At high velocity the edge of the flattened drop destabilizes during the recession into many small azimuthal droplets.

Above the surface, at moderate We, a silicone oil jet is ejected into the air, as in Fig. 2(b). At larger We, a silicone oil sheet, initially covered by the flattened drop, is ejected at the periphery of the crater. Above $\mathrm{We} \sim 10^{3}$, the sheet destabilizes and eventually fragments. The drop recession is, however, faster than this destabilization, and the drop fragmentation is essentially not influenced by that of the sheet.

Figure 2 shows the number of daughter drops $N$ generated per impact for increasing We together with the separations between the three fragmentation regimes. $N$ is obviously equal to one in regime 1 . It globally increases, although nonmonotonically, with respect to We up to typically ten in regime 2 and then increases regularly and strongly with We in regime 3 (a dependency we explain below) to reach 300 daughter drops per impact at $\mathrm{We}=1250$, the limit in our experimental setup.

Regime 2 is due to the breakup of the ligament-shaped drop by a Plateau-Rayleigh mechanism. The number of drops produced depends in a discrete but reproducible way on which particular oscillation mode of the drop is excited; this is why $N$ does not increase monotonically with

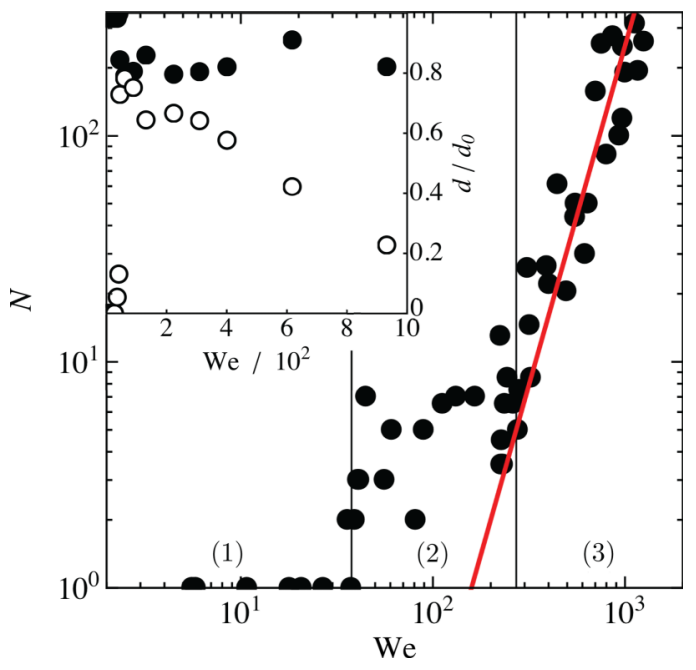

FIG. 2 (color online). Number of drops $N$ produced per impact versus Weber number We, for $\nu_{B}=10 \mathrm{~mm}^{2} \mathrm{~s}^{-1}$. The black vertical lines separate the no-fragmentation regime (1), the axial (2), and azimuthal (3) fragmentation regimes. The red line is $N \sim \mathrm{We}^{3}$ according to equation (2). Inset: Diameter $d$ of the largest (filled circle) and second largest daughter drops (hollow circle).
We in this regime (by contrast with regime 3 where the nonmonotonic increase in $N$ is due to small random scatter). The ligament formation is a consequence of the flow-focussing on the axis of symmetry during the crater collapse. We will not concentrate here on this fragmentation regime, but only remark that it shows similarities with the breakup of the central jet ejected for miscible drop impact [2] or bursting of surface bubbles [17]. We indeed observe in our experiments that, in regime 2, not all daughter drops stay in the bath: Some are actually ejected above the surface into the atmosphere. A similar fragmentation regime, i.e., with axial breakup after radial recession, is also observed for drops impacting on solid hydrophobic surfaces (see for instance images in [18]).

Regime 3, on the other hand, is due to the azimuthal destabilization of the retracting flattened drop edge which is illustrated in Fig. 3. Here also, the process shows common features with miscible drop impact where the liquid sheet which is ejected around the crater develops a rim which is likely to destabilize in the azimuthal direction, the so-called crown, and to fragment into small droplets. Reference [4] attributed this fragmentation to the PlateauRayleigh breakup of the toroidal rim. A similar pattern is also observed for impact on solid surfaces [14] where the fragmentation of the rim bordering the flattened drop is attributed to a Rayleigh-Taylor [19-21] mechanism. This mechanism should however have less influence here, since the density difference between the drop and the bath is relatively much smaller than for a drop receding into air.

Having reported the different fragmentation behaviors, we now focus on regime 3 , relevant for high velocity impacts and large numbers of daughter drops. In order to elucidate the fragmentation dynamics and its products, we

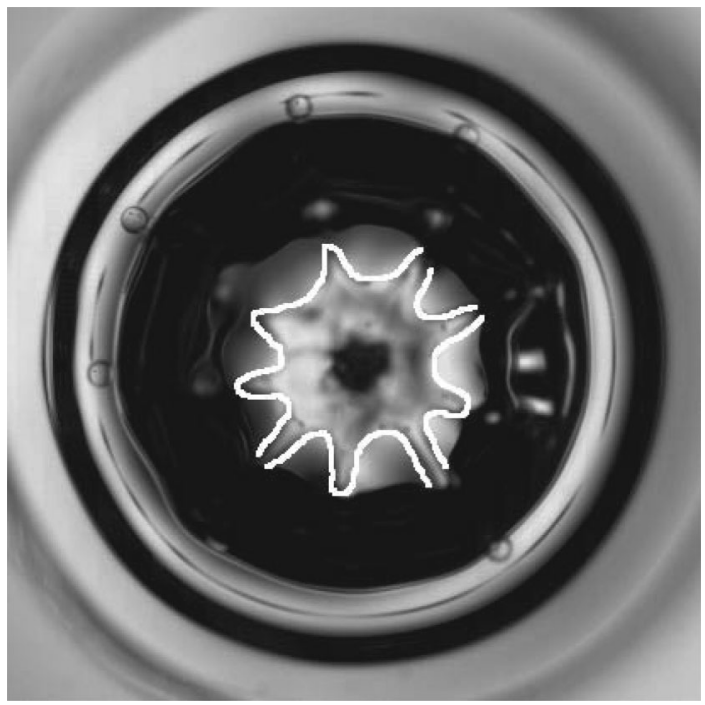

FIG. 3. Bottom view of the flattened drop recession after impact emphasizing the azimuthal destabilization of the receding drop edge. The white line follows the drop contour. 
measured the spreading of the drop at impact. Figure 4 shows the maximal area $\Sigma_{m}$ covered by the drop onto the crater surface (see Fig. 1) normalized by the impacting drop cross section $\Sigma_{0}=\pi d_{0}^{2} / 4$ versus We. It is measured from the side view assuming cylindrical symmetry of the flattened drop, which holds before fragmentation begins. For each bath viscosity we observe that $\Sigma_{m}$ increases as

$$
\Sigma_{m} / \Sigma_{0}-1 \propto \mathrm{We}
$$

with a proportionality factor slowly decreasing with increasing bath viscosity and possibly depending on the balance between $\sigma, \sigma_{B}$, and $\sigma_{D B}$. This means that for a given bath viscosity the same fraction of the drop kinetic energy $\pi \rho V^{2} d_{0}^{3} / 12$ is converted into the surface energy $\left(\sigma+\sigma_{D B}-\sigma_{B}\right) \Sigma_{m}$ of the flattened drop. It equivalently means that the energy dissipated during the spreading of the drop is proportional to $\Sigma_{m}$. This dissipation is certainly localized in, or closely around, the drop since we measured that the sum of the surface energy, the bath gravity potential energy, and the energy dissipated in the far flow represents less than $50 \%$ of the initial kinetic energy of the drop for We $\gtrsim 100$ (see Supplemental Material B [13]). For small bath viscosity, the discrepancy between Eq. (1) and the measurements at large We is a signature of the onset of the azimuthal fragmentation regime (and also of the extra dissipation associated with the chaotic motion observed for the largest Reynolds numbers $d_{0} V / \nu_{B} \simeq 2 \times 10^{4}$ reached with $\nu_{B}=0.65 \mathrm{~mm}^{2} \mathrm{~s}^{-1}$ ). Indeed, the smaller the viscosity, the smaller the critical Weber number for fragmentation as the inset of Fig. 4 reveals.

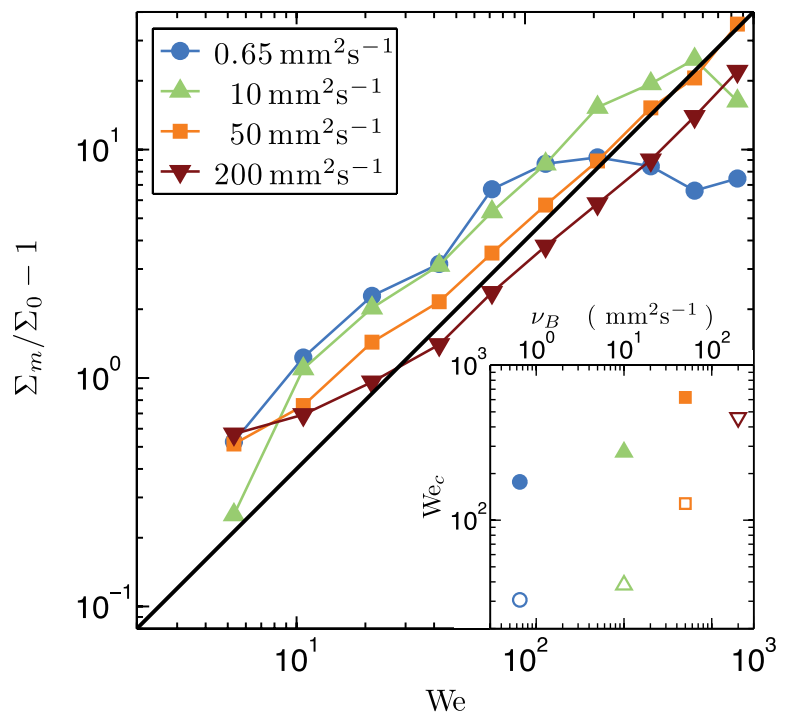

FIG. 4 (color online). Maximal area $\Sigma_{m}$ covered by the flattened drop versus We for increasing bath viscosities $\nu_{B}$. The black line has slope one. Inset: Threshold Weber number for axial fragmentation (hollow symbols) and for azimuthal fragmentation (filled symbols) regimes. $\mathrm{We}_{c}>1250$ for $\nu_{B}=200 \mathrm{~mm}^{2} \mathrm{~s}^{-1}$.
The maximal spreading $\Sigma_{m}$ has important consequences on the drop's subsequent fragmentation. During the recession of the flattened drop the drop liquid is progressively collected at the edge. The edge develops azimuthal modulations which are eventually torn outward into radial ligaments as a consequence of the edge inward motion. The ligaments then pinch off by a Plateau-Rayleigh mechanism to form the population of daughter drops as illustrated in Fig. 1. Similar configurations leading to identical destabilization patterns are observed for the bursting of water films in a viscous environment [22], the recession of a water film between colliding viscous droplets [23] or the rising of a bubble through the interface between two immiscible liquids [24]. References [22,23] concluded that, although the viscous drag is involved in the tearing of the ligaments, the instability of the edge at the root of the drop fragmentation is of a PlateauRayleigh type.

In our experiments at large We the flattened drop edge permanently destabilizes and empties into the radial ligaments while receding (see Fig. 3). Direct observations suggest that the ligament radius $r$ is always comparable with the current drop thickness $h$ and we therefore assume $r \sim h$. Since the ligaments' breakup time is of the same order as the typical evolution time of the recession (see Supplemental Material C [13]), the ligaments pinch off before they are substantially stretched. Then, according to the Plateau-Rayleigh paradigm the most unstable wavelength of the pinch-off is $\lambda \simeq 9 r$ [25], which together with $r \sim h$ and mass conservation yields for the daughter drops diameter $d \sim h$. Assuming now that the flattened drop has a uniform thickness $h$, at maximal spreading $h=h_{m} \sim$ $d_{0}^{3} / \Sigma_{m}$ from mass conservation. Considering lastly that a non-negligible portion of the impacting drop fragments, the typical number of daughter drops is

$$
N \sim d_{0}^{3} / d^{3} \sim d_{0}^{3} / h^{3} \sim \Sigma_{m}^{3} / d_{0}^{6} \sim \mathrm{We}^{3},
$$

by making use of (1) in the limit $\Sigma / \Sigma_{0} \gg 1$. This scaling law for $N$ is shown in Fig. 2. It is in good agreement with measurements for high We, i.e., for the regime 3 we are discussing now.

The same assumption $r \sim h$ can also explain the structure of the daughter drops population, i.e., the dispersion in their diameters $d$. For the high We $\left(\gtrsim 10^{2}\right)$ we are considering, $h$ is much smaller than the typical size of the crater and we can therefore consider it as a disk with time-dependent area $\Sigma$. This cylindrical drop fragments at its edge while receding. Our measurements of the size of the largest and second largest daughter drops (inset of Fig. 2) show that if the latter is decreasing with We as one expects, the former is by contrast rather constant at approximately $0.8 d_{0}$. This means that although the impacting drop may fragment at its edge into a few hundred drops, a substantial fraction of it recedes to the axis of symmetry to form one large daughter drop as one can also see in 


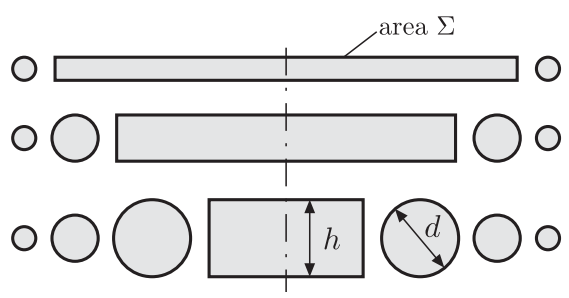

FIG. 5. Mechanism explaining the broad distribution of daughter drop sizes. Time increases from top to bottom.

Fig. 1. Based on these observations, we propose a simple model for the daughter drop sizes as schematized in Fig. 5. We assume that $h$ remains uniform over the drop during the recession and that only a small fraction of the drop actually fragments, i.e., $h \simeq d_{0}^{3} / \Sigma$. Writing that each daughter drop has a volume $\sim d^{3}$, mass conservation relates the variation $d N$ in the number of daughter drops during the recession with that in the flattened drop area $d \Sigma$ according to $d^{3} d N \sim$ $h d \Sigma$. Using now $d \sim h$ one obtains by variable substitution

$$
d N(d) \sim \frac{d_{0}^{3}}{d^{4}} d d,
$$

which simply means that many small droplets are formed at the early stage of the recession when the drop is thin and widely spread, while only a few ones are formed at the late stage when the drop is thick and compact, a daughter drop hierarchy consistent with our observations but also with those of [24] for a similar fragmentation configuration. Equation (3) is expected to be valid between the minimal drop size

$$
d_{\min } \sim d_{0}^{3} / \Sigma_{m} \sim d_{0} / \mathrm{We}
$$

observed for the maximal spreading of the drop, and the maximal size of order $d_{0}$, when the flattened drop has receded to the axis. Taking into account this range of diameters, the distribution of drop sizes $d N / d d$ [from equation (3)] can be written in normalized form as

$$
p(x=d /\langle d\rangle)=\frac{8}{9} \frac{\left(d_{\min }^{-3}-d_{0}^{-3}\right)^{2}}{\left(d_{\min }^{-2}-d_{0}^{-2}\right)^{3}} x^{-4} \simeq \frac{8}{9} x^{-4}
$$

where $\langle d\rangle$ is the mean diameter of the daughter drops. The simplification comes from $d_{\min } / d_{0} \ll 1$. With this step the distribution becomes independent of the upper bound $d_{0}$. When normalized by $\langle d\rangle \sim d_{\min }$ the probability density function therefore adopts a universal shape that, remarkably, is independent of $d_{\min }$, i.e., independent of We.

Figure 6 shows a comparison of Eq. (5) with measurements of the daughter drop size distribution for four different We in regime 3, ranging from 550 to 1170 . The size distributions are measured by taking high resolution pictures through the clean transparent bottom of the container after all the daughter drops (denser than the liquid bath)

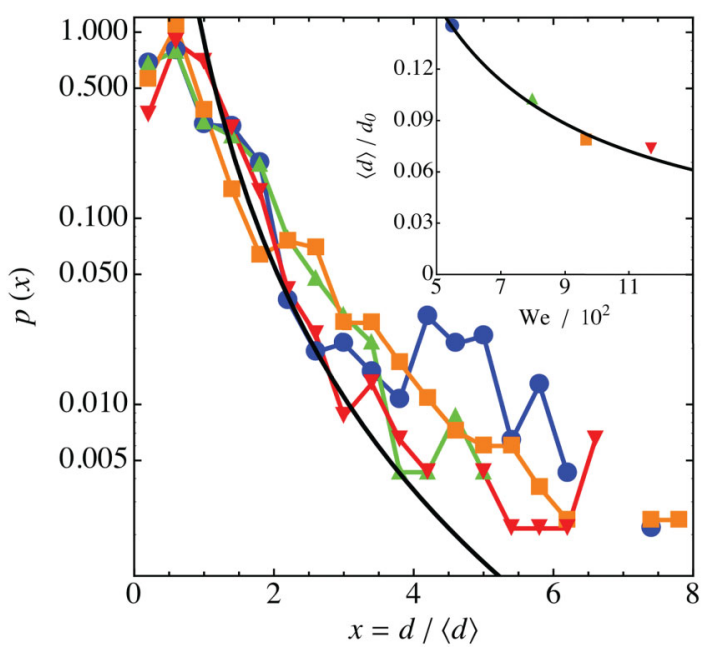

FIG. 6 (color online). Normalized distribution functions of the daughter drop diameters $d$ for $\mathrm{We}=550$ (blue circles), 800 (green triangles), 970 (orange squares), and 1170 (red inverted triangles) with $\nu_{B}=10 \mathrm{~mm}^{2} \mathrm{~s}^{-1}$. The black line is $p(x)=(8 / 9) x^{-4}$ from equation (5). Inset: Mean drop diameter $\langle d\rangle$. The black line is $\langle d\rangle \propto \mathrm{We}^{-1}$ according to (2).

deposited onto it (see Supplemental Material D [13]). Each of them, being based on more than one thousand drops, is statistically converged. They reveal several features. First, the mean size $\langle d\rangle$ is proportional to $\mathrm{We}^{-1}$ (see inset) in agreement with (2). Second, the peaks for small dimensionless diameters $d /\langle d\rangle$ overlap for different Weber numbers showing that $d_{\min } \sim\langle d\rangle$ in agreement with (2) and (4). Third, although the size distribution (5) is not able to capture the discrete influence of the largest drops for moderate $\mathrm{We}$, since it is by essence continuous, it is a good model for high We when the discrete features can be forgotten due to the large number of daughter drops.

Finally, let us remark that, although similarities in the fragmentation patterns are observed, the dynamics presented here differs from that of the impact onto a solid. Due to the liquid bath deformation and its wetting of water, the drop essentially recedes into the silicone oil and not into air, which might significantly influence the destabilization mechanism at the drop's edge as observed in [22-24]. The impact onto a solid can certainly be considered as the limiting case of an infinite bath viscosity. One therefore expects that the threshold Weber number for azimuthal fragmentation will reach a constant value for large viscosities that corresponds to the threshold for the impact onto a solid. As we could check, this latter threshold, however, depends strongly on the contact angle $\theta$ of the drop on the solid (varying between approximately 100, for a water drop in the Leidenfrost state with $\theta=180^{\circ}$, and more than 1250 , for a water drop impacting onto a glass plate with $\theta \simeq 30^{\circ}$ ). The transition to the solid limit, and the influence of the contact angle on the fragmentation are therefore important unsolved questions that would both deserve an independent study. 
*h.lhuissier@utwente.nl

[1] A. M. Worthington, Proc. R. Soc. A 34, 217 (1882).

[2] A. M. Worthington and R. S. Cole, Phil. Trans. R. Soc. A 189, 137 (1897).

[3] O. Engel, J. Appl. Phys. 37, 1798 (1966).

[4] L. Zhang, P. Brunet, J. Eggers, and R. Deegan, Phys. Fluids 22, 122105 (2010).

[5] L. Xu, W. W. Zhang, and S. R. Nagel, Phys. Rev. Lett. 94, 184505 (2005).

[6] L. Zhang, J. Toole, K. Fezzaa, and R. Deegan, J. Fluid Mech. 690, 5 (2011).

[7] L. Esmailizadeh and R. Messler, J. Colloid Interface Sci. 110, 561 (1986).

[8] Q. Deng, V. Anilkumar, and T. Wang, J. Fluid Mech. 578, 119 (2007).

[9] A. Prosperetti and H. Oguz, Annu. Rev. Fluid Mech. 25, 577 (1993).

[10] A. J. Yule and J.J. Dunkley, Atomization of Melts (Clarendon Press, Oxford, 1994).

[11] S. Nashimura, Z.-G. Zhang, K.-I. Sugiyama, and I. Kinoshita, Nucl. Eng. Des. 237, 2201 (2007).

[12] N. Bremond, E. Santanach-Carreras, L.-Y. Chu, and J. Bibette, Soft Matter 6, 2484 (2010).

[13] See Supplemental Material at http://link.aps.org/ supplemental/10.1103/PhysRevLett.110.264503 for the properties of the liquids, the calculation of the energy dissipated in the far field, the typical ligaments' breakup time scale and the method for the daughter drops size measurements.

[14] A. M. Worthington, Proc. R. Soc. London 25, 261 (1876).

[15] S. Chandra and C. T. Avedisian, Proc. R. Soc. A 432, 13 (1991).

[16] G. Delon, D. Terwagne, S. Dorbolo, N. Vandewalle, and H. Caps, Phys. Rev. E 84, 046320 (2011).

[17] F. H. Knelman, N. Dombrowski, and D. M. Newitt, Nature (London) 173, 261 (1954).

[18] D. Richard, C. Clanet, and D. Quéré, Nature (London) 417, 811 (2002).

[19] A. Rozhkov, B. Prunet-Foch, and M. Vignes-Adler, Proc. R. Soc. A 460, 2681 (2004).

[20] N. Mehdizadeh, S. Chandra, and J. Mostaghimi, J. Fluid Mech. 510, 353 (2004).

[21] E. Villermaux and B. Bossa, J. Fluid Mech. 668, 412 (2011).

[22] E. Reyssat and D. Quéré, Europhys. Lett. 76, 236 (2006).

[23] H. Aryafar and H. P. Kavehpour, Phys. Rev. E 78, 037302 (2008).

[24] T. Uemura, Y. Ueda, and M. Iguchi, Europhys. Lett. 92, 34004 (2010).

[25] J. W. Lord Rayleigh, Proc. London Math. Soc. s1-10, 4 (1878). 